Case Report

\title{
Diagnosis, PET/CT imaging, and treatment of extranodal non-Hodgkin lymphoma in keratinized gingiva: a case report
}

\author{
Cüneyt A. Aral1), Osman S. Ağlarc12), Hasan H. Yılmaz'), Funda Taşl13), \\ Serap Karaarslan ${ }^{3)}$, Filiz Hatipoğlu4), and Mustafa S. Sanal ${ }^{5)}$ \\ 1)Department of Periodontology, Faculty of Dentistry, Sifa University, Izmir, Turkey \\ 2)Department of Oral and Maxillofacial Radiology, Faculty of Dentistry, Sifa University, Izmir, Turkey \\ 3)Department of Pathology, Faculty of Medicine, Sifa University, Izmir, Turkey \\ 4)Department of Nuclear Medicine, Faculty of Medicine, Sifa University, Izmir, Turkey \\ 5)Private Practice, Department of Oncology, Tinaztepe Hospital, Izmir, Turkey
}

(Received September 23, 2014; Accepted January 31, 2015)

\begin{abstract}
A 58-year-old patient who smoked and had uncontrolled type 2 diabetes mellitus was referred to our clinic. The patient had a suspicious asymptomatic lesion that was diagnosed as B-cell non-Hodgkin lymphoma (NHL). Immunohistochemistry revealed intense and diffuse expression of CD20, CD10, BCL-6, and Ki-67. A positron emission tomography/ computed tomography (PET/CT) scan showed focal pathological uptake of F-18-fluorodeoxyglucose only in the subcutaneous tissue anterior to the left maxillary sinus. After lesion excision and five courses of chemotherapy, PET/CT scans demonstrated complete resolution of the lesion. Smoking, uncontrolled diabetes mellitus, and periodontal disease might be predisposing factors for oral NHL.

(J Oral Sci 57, 59-62, 2015)
\end{abstract}

Keywords: gingiva; histopathology; non-Hodgkin

lymphoma; positron emission tomography.

\section{Introduction}

Lymphoma, which is characterized by proliferation of lymphoid cells, is a heterogeneous malignancy. In general, lymphoma is classified as either Hodgkin's

Correspondence to Dr. Cüneyt Asım Aral, Department of Periodontology, Faculty of Dentistry, Sifa University, 35100 Izmir, Turkey

Fax:+90-232-3435656 E-mail: cuneytasimaral@gmail.com

doi.org/10.2334/josnusd.57.59

DN/JST.JSTAGE/josnusd/57.59 or non-Hodgkin lymphoma (NHL). While Hodgkin's lymphoma is predominantly a nodal-based disease, nearly one-third of NHL forms in tissues other than the lymph nodes (1). Primary malignant lymphoma in the oral cavity is rare (2). NHL of the oral cavity and oropharynx is defined as either B- or natural killer (NK)/T-lymphoid cell cancer. Bone involvement also occurs in some rare cases (3). Oral lesions most frequently present as painless erythematous enlargements that may become ulcerated and hemorrhagic because of traumatic injury.

Immunohistochemical examination and novel imaging methods such as positron emission tomography/ computed tomography (PET/CT) have been shown to enable successful diagnosis and management of NHL $(3,4)$. However, the application of PET/CT has not been reported for clinical diagnosis of primary NHL in the gingiva. The present report highlights the etiology, diagnosis, imaging, and treatment of NHL in the gingiva that mimicked a periodontal infection.

\section{Case Report}

A 58-year-old Caucasian male presented with a 2-month history of relatively rapid growth of a mass in the keratinized gingiva of his maxillary anterior teeth. The patient was referred to our clinic for consultation after a potential diagnosis of periodontal disease. The patient had type 2 diabetes mellitus and was under oral anti-diabetic agents for the previous 8 years. He had been smoking approximately 20 cigarettes a day for the previous 20 years.

Physical and extraoral examinations of the patient were normal. Intraoral examination included dental, peri- 


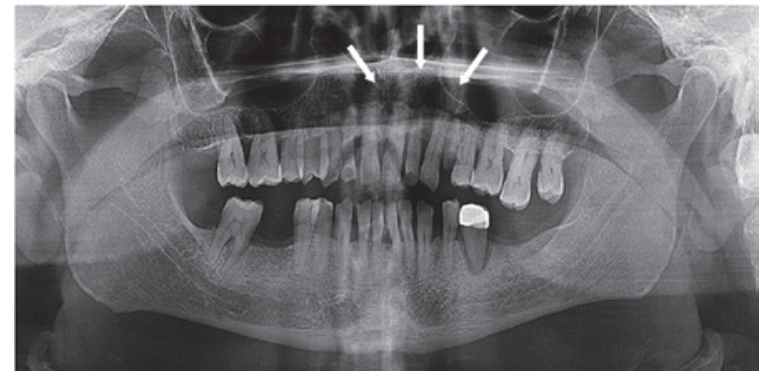

Fig. 1 A panoramic radiograph was scanned and digitized to enhance the visualization of the patient's baseline periodontal status. The radiograph shows generalized horizontal and vertical bone loss in all quadrants. Arrows indicate the localization of the lesion.
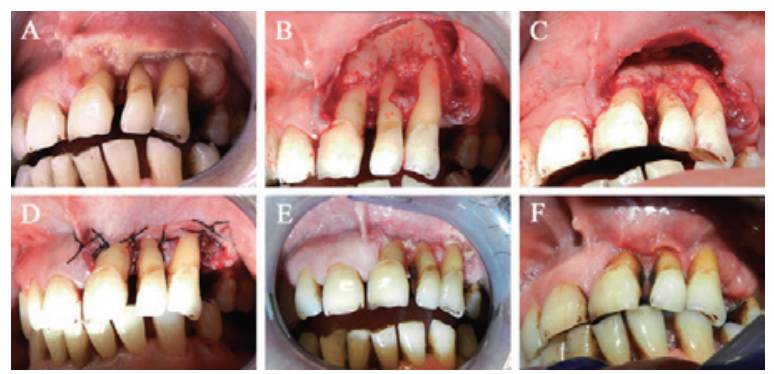

Fig. 2 Non-Hodgkin's lymphoma of the anterior maxillary gingiva. Intra- and post-operative clinical photographs (A-F). A solitary lesion was seen in the maxillary anterior region which was slightly elevated, and had localized necrotic ulcerative areas (A). An excisional biopsy of the gingiva was performed, and all remaining granulation tissue was removed (B and C). The flap was coronally advanced, and primary closure was achieved with 3.0 silk sutures (D). Images illustrate the healing process on post-operative days $10(\mathrm{E})$ and $30(\mathrm{~F})$.

odontal, oral, and pharyngeal status. Generalized alveolar bone loss was observed on the panoramic radiograph (Fig. 1). A solitary lesion was seen around the maxillary anterior tooth which was slightly elevated and showed no hyperpigmentation, discoloration, or spontaneous bleeding. The lesion had localized necrotic ulcerative areas. Application of gentle pressure to the lesion caused pus to appear throughout the gingival sulcus of adjacent teeth. The patient did not experience pain from a percussion test or during chewing as a result of the affected teeth. The periodontal condition of the teeth associated with the NHL lesion was determined by gingival index (GI), probing depth (PD), distance from cemento-enamel junction (CEJ) to gingival margin (GM), clinical attachment level (CAL), and mobility scores (5). A periodontal probe was used to measure 6 sites per tooth: 1) mesiobuccal; 2) mid-buccal; 3) disto-buccal; 4) mesio-lingual; 5) mid-lingual; and 6) disto-lingual. Mobility of the adjacent teeth 21, 22, and 23 was increased, and severe clinical attachment loss was noted (Table 1).

The lesion was suspected to be precancerous, and the
Table 1 Periodontal characteristics of the teeth associated with non-Hodgkin lymphoma

\begin{tabular}{|c|c|c|c|c|c|c|}
\hline & \multicolumn{2}{|c|}{ Tooth \#21 } & \multicolumn{2}{|c|}{ Tooth \#22 } & \multicolumn{2}{|c|}{ Tooth \#23 } \\
\hline & Facial & Palatal & Facial & Palatal & Facial & Palatal \\
\hline GI & 223 & 112 & 232 & 222 & 223 & 222 \\
\hline $\mathrm{PD}(\mathrm{mm})$ & 223 & 222 & 434 & 323 & 244 & 224 \\
\hline CEJ-GM (mm) & 136 & 125 & 765 & 664 & 476 & 344 \\
\hline CAL (mm) & 359 & 347 & 1199 & 987 & 61110 & 568 \\
\hline Mobility & & & 2 & 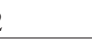 & c & 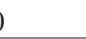 \\
\hline
\end{tabular}

Periodontal condition of the teeth associated with non-Hodgkin lymphoma, as determined by gingival index (GI), probing depth (PD), distance from cemento-enamel junction (CEJ) to gingival margin (GM), and clinical attachment level (CAL). A periodontal probe was used to measure 6 sites per tooth: 1) mesio-buccal; 2) mid-buccal; 3) distobuccal; 4) mesio-lingual; 5) mid-lingual; and 6) disto-lingual.
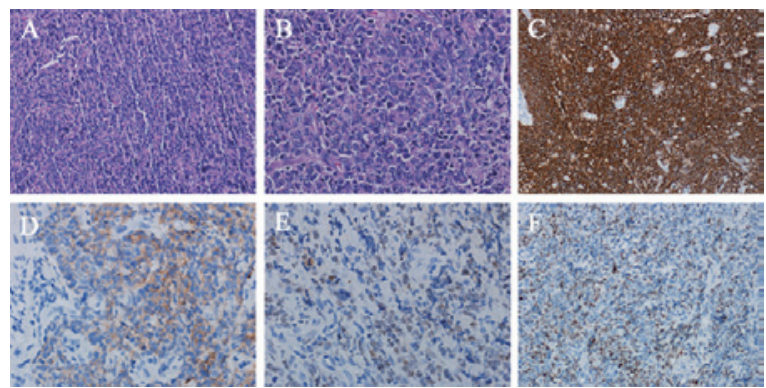

Fig. 3 Histopathological examination of the excised lesion. Diffuse infiltration of medium- to large-sized tumor cells with marked nucleii and a small amount of basophilic cytoplasm (A; H\&E stain, $\times 20$ magnification). Tumor cells with remarkable nucleii and apoptotic substances (B; H\&E stain, $\times 40$ magnification). Immunohistochemical staining of tumor cells is shown in C to F. Diffuse CD20(+) cells (C; CD20, $\times 20$ magnification). Focal CD10 $(+)$ cells $(\mathrm{D} ; \mathrm{CD} 10, \times 40$ magnification). Several BCL-6(+) cells (E; BCL-6, $\times 40$ magnification). Ki-67(+) cells (F; Ki-67, ×20 magnification).

patient was recommended for medical consultation. The patient's fasting glucose $(253 \mathrm{mg} / \mathrm{dL})$, and hemoglobin A1c (8.72) levels were high. Systematic serological and blood viral tests were negative. The patient's hemogram was within normal limits with no signs of inflammation.

After medical consultation, excisional biopsy of the gingiva was performed (Fig. 2A-C). Subsequently, granulation tissues on the alveolar bone were removed and scaling and root planing were performed. The flap was coronally advanced, and primary closure was achieved with 3.0 silk sutures (Fig. 2D). Post-operative amoxicillin/clavulanic acid (1,000 $\mathrm{mg}$ twice a day), flurbiprofen (100 mg twice a day), and chlorhexidine gluconate $(0.12 \%$ twice a day) were prescribed. Oral hygiene instructions were given to the patient before the surgery. The patient was instructed to use an extra soft toothbrush and soft interdental brushes in the anterior teeth of the affected area. The patient was encouraged to cease smoking before and after the surgery. The patient was followed-up on postoperative days 10 (Fig. 2E) and 30 (Fig. 2F). 

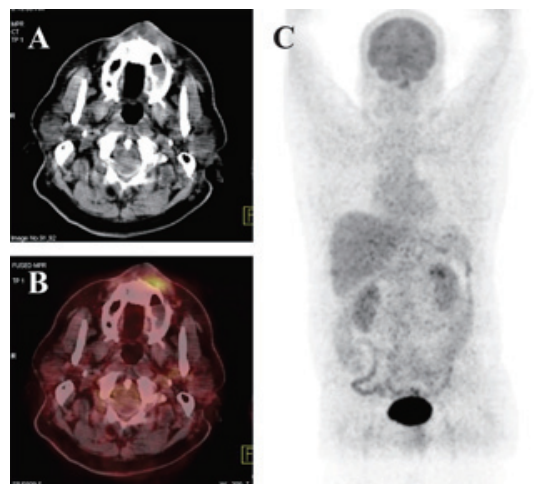

Fig. 4 Images A, B, and C show axial computed tomography (CT), fused positron emission tomography (PET)/CT, and maximum intensity projection images, respectively. Axial CT (A) and fused PET/CT (B) show moderate tracer uptake with a maximum standardized uptake value $\left(\mathrm{SUV}_{\max }\right)$ of 4.4. A PET/CT image illustrates the involvement of subcutaneous tissue anterior to the maxillary sinus left of the midline. There was no other pathological uptake of F-18-fluorodeoxyglucose in the rest of the body $(\mathrm{C})$.

Table 2 Periodontal characteristics of the teeth associated with non-Hodgkin lymphoma 16 months post-operatively

\begin{tabular}{|c|c|c|c|c|c|c|}
\hline & \multicolumn{2}{|c|}{ Tooth \#21 } & \multicolumn{2}{|c|}{ Tooth \#22 } & \multicolumn{2}{|c|}{ Tooth \#23 } \\
\hline & Facial & Palatal & Facial & Palatal & Facial & Palatal \\
\hline GI & $\begin{array}{lll}1 & 1 & 1\end{array}$ & $\begin{array}{lll}1 & 0 & 1\end{array}$ & $\begin{array}{lll}111 & 1\end{array}$ & 222 & 112 & $\begin{array}{lll}0 & 0 & 0\end{array}$ \\
\hline $\mathrm{PD}(\mathrm{mm})$ & 222 & 212 & 212 & 222 & 212 & 222 \\
\hline CEJ-GM (mm) & 147 & 135 & 885 & 664 & 497 & 346 \\
\hline CAL (mm) & 369 & 347 & 1097 & 886 & 6109 & 568 \\
\hline Mobility & & & & 2 & & \\
\hline
\end{tabular}

Abbreviations: gingival index (GI), probing depth (PD), distance from cemento-enamel junction (CEJ) to gingival margin (GM), and clinical attachment level (CAL).

Pathological examination of the enucleated tissue indicated that the stroma was densely infiltrated by lymphoma cells that were primarily established around blood vessels and that had razed all the normal healthy tissues. Immunohistochemical analysis revealed intense and diffuse CD20 expression, focal CD10 expression, a few BCL-6 positive cells, and a small number of Ki-67 stained cells with a proliferative index of $30 \%$ (Fig. 3C-F). Moreover, immunohistochemical staining of the lesion was negative for cyclin D1, BCL-2, CD23, CD30, CD138, ALK, CD5, and pansitokeratin. Therefore, the lesion was diagnosed as B-cell NHL.

To examine the possibility that the tumor arose as a metastasis, the patient underwent a bone marrow aspiration biopsy. The bone marrow samples were normal. Next, full body PET/CT scans were performed 60 minutes after intravenous injection of $555 \mathrm{MBq}$ F-18-fluorodeoxyglucose (FDG). Images were acquired in three dimensions from the vertex to the upper thigh with the patient in a supine position with his arms raised over his head (Fig. 4). Whole body CT images were acquired, and
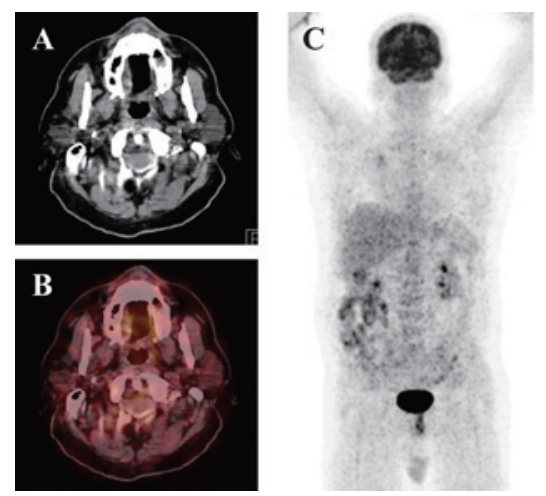

Fig. 5 Images A, B, and C illustrate axial computed tomography (CT), fused positron emission tomography (PET)/CT, and maximum intensity projection images, respectively. A PET/CT scan after chemotherapy shows complete remission. There was no hypermetabolic focus within the body.
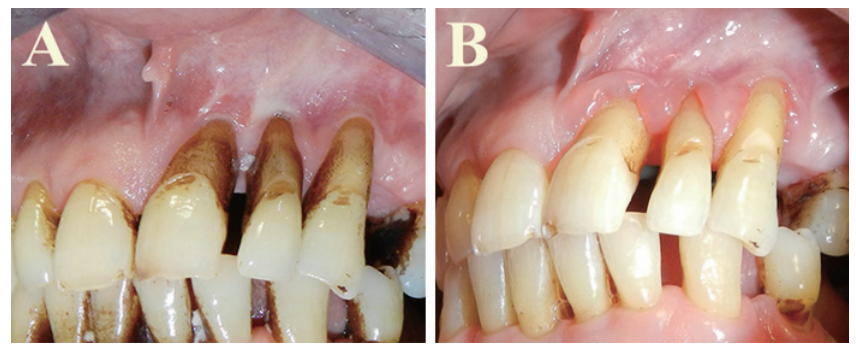

Fig. 6 Intraoral pictures show the clinical health of periodontal tissues 6 months (A) and 16 months (B) post-operatively. No clinical signs of NHL progression in the gingiva were noted.

intravenous contrast was not used during scanning. The acquired data was reconstructed using CT data attenuation correction. The CT, PET, and co-registered PET/CT images were reviewed in transaxial coronal and sagittal planes along with maximum-intensity projection of whole body coronal images.

An oncologist found that the systemic screen for metastases of the lymphoma was negative. PET/CT scans revealed subcutaneous tissue involvement anterior to the maxillary sinus, left of the midline. The tumor was classified as stage IE, with no systemic symptoms, according to the Ann Arbor Staging Classification (6). The patient received 5 cycles of R-CHOP (rituximab, cyclophosphamide, doxorubicin, vincristine, and prednisolone). To ensure complete long-term resolution of the NHL, PET/CT scans were repeated after 3 months of chemotherapy. The PET/CT results showed no recurrent lesions within the body (Fig. 5). Chemotherapy resulted in complete remission of the NHL. No clinical signs of NHL progression in the gingiva were observed during 16 months of remission, as seen in Fig. 6. The patient's 
periodontal condition improved after 16 months of postoperative maintenance (Table 2).

\section{Discussion}

An association between NHL and diabetes mellitus has been reported (7). Moreover, cigarette smoking is associated with an increased risk of NHL (8). In the current case, the pathogenesis of NHL may be due to diabetes mellitus and smoking. Additionally, a report indicated that NHL might occur in healing sockets after extraction of teeth due to periodontal disease (9). Therefore, in addition to diabetes mellitus and smoking, periodontitis may have played a role in the etiology of NHL in this case.

Oral NHL is not frequently seen in the clinic, and its clinical features can be confused for a vast range of periodontal diseases. In order to provide the correct treatment, differential diagnosis between benign and malignant lesions should be considered.

Squamous cell carcinoma accounts for $95 \%$ of all cancers of the oral cavity. The second most common cancer of the oral cavity is NHL, which accounts for $3.5 \%$ of all oral malignancies (10). Therefore, it is particularly important to distinguish NHL from squamous cell carcinoma. Hodgkin's lymphoma, acute and chronic leukemia, and metastatic tumors should also be differentially diagnosed.

PET is a novel imaging technique that produces three-dimensional images of functional processes in the body. PET, which has become an established nuclear imaging modality, uses compounds labeled with positron emitting radioisotopes as molecular probes that are introduced into the body on a biologically active molecule. Three-dimensional images of the concentration of positron-emitting radionuclides within the body are then constructed by computer analysis. PET is especially beneficial in oncology where it is used to identify metastasis, establish prognosis, and assess response to therapy of NHL (4). To the best of our knowledge, this is the first case of PET imaging of NHL in the gingiva. In this report, baseline and post-treatment PET/CT images revealed that complete remission of NHL was achieved by excision of the tumor and chemotherapy.

In conclusion, the occurrence of NHL in keratinized gingiva is extremely rare. However, clinicians should always be aware of such malignant lesions, which can spread in oral tissues.

The clinical features of NHL mimic other pathological entities of the oral cavity, and treatment of these malignancies may be delayed because of misdiagnosis. Therefore, it is important to examine suspected lesions extensively and to confirm diagnoses with tissue biop- sies. Furthermore, PET/CT imaging is beneficial in the diagnosis, staging, management, and evaluation of treatment efficacy for oral NHL.

There is also a great need for prolonged care of patients following diagnosis. Our observations underscore the importance of preservation of periodontal tissues by smoking cessation, controlling periodontal disease, and management of type 2 diabetes mellitus so that these risk factors that may contribute to the pathogenesis of these malignant lesions can be excluded.

\section{References}

1. Triantafillidou K, Dimitrakopoulos J, Iordanidis F, Gkagkalis A (2012) Extranodal non-Hodgkin lymphomas of the oral cavity and maxillofacial region: a clinical study of 58 cases and review of the literature. J Oral Maxillofac Surg 70, 27762785.

2. Boulaadas M, Benazzou S, Sefiani S, Nazih N, Essakalli L, Kzadri M (2008) Primary extranodal non-Hodgkin lymphoma of the oral cavity. J Craniofac Surg 19, 1183-1185.

3. Navarro CM, Shibli JA, Ferrari RB, d'Avila S, Sposto MR (2008) Gingival primary extranodal non-Hodgkin's lymphoma as the first manifestation of acquired immunodeficiency syndrome. J Periodontol 79, 562-566.

4. Dunleavy K, Mikhaeel G, Sehn LH, Hicks RJ, Wilson WH (2010) The value of positron emission tomography in prognosis and response assessment in non-Hodgkin lymphoma. Leuk Lymphoma 51, Suppl 1, 28-33.

5. Schellinck AE, Rees TD, Plemons JM, Kessler HP, Rivera-Hidalgo F, Solomon ES (2009) A comparison of the periodontal status in patients with mucous membrane pemphigoid: a 5-year follow-up. J Periodontol 80, 17651773.

6. Jones SE, Fuks Z, Bull M, Kadin ME, Dorfman RF, Kaplan HS et al. (1973) Non-Hodgkin's lymphomas. IV. Clinicopathologic correlation in 405 cases. Cancer 31, 806-823.

7. Castillo JJ, Mull N, Reagan JL, Nemr S, Mitri J (2012) Increased incidence of non-Hodgkin lymphoma, leukemia, and myeloma in patients with diabetes mellitus type 2: a meta-analysis of observational studies. Blood 119, 48454850.

8. Morton LM, Hartge P, Holford TR, Holly EA, Chiu BC, Vineis P et al. (2005) Cigarette smoking and risk of nonHodgkin lymphoma: a pooled analysis from the International Lymphoma Epidemiology Consortium (interlymph). Cancer Epidemiol Biomarkers Prev 14, 925-933.

9. Hokett SD, Cuenin MF, Peacock ME, Thompson SH, Van Dyke TE (2000) Non-Hodgkin's lymphoma and periodontitis. A case report. J Periodontol 71, 504-509.

10. Epstein JB, Epstein JD, Le ND, Gorsky M (2001) Characteristics of oral and paraoral malignant lymphoma: a population-based review of 361 cases. Oral Surg Oral Med Oral Pathol Oral Radiol Endod 92, 519-525. 